\title{
COPD - Diese Faktoren bestimmen die Therapie
}

In den aktualisierten Leitlinien der Global Initiative For Chronic Obstructive Lung Disease (GOLD) wurden die Empfehlungen zur medikamentösen Therapie der COPD deutlich vereinfacht. Für jeden Schweregrad wurde ein entsprechender Algorithmus festgelegt.

In Deutschland leiden etwa drei bis fünf Millionen Menschen an einer chronisch obstruktiven Lungenerkrankung (COPD). Da Tabakrauch der wichtigste Risikofaktor für die Entstehung einer COPD ist, ist die Raucherentwöhnung nach der aktualisierten Leitlinie der Global Initiative For Chronic Obstructive Lung Disease (GOLD) die wirksamste Maßnahme, mit der sich der natürliche Verlauf einer COPD beeinflussen lässt.

Ihre Empfehlungen zur medikamentösen Behandlung von COPD-Patienten haben die Autoren der neuen GOLDLeitlinie vereinfacht. So werden für die ABCD-Einteilung, auf der die medikamentöse Therapie basiert, jetzt nur noch die Symptome und das Exazerbationsrisiko berücksichtigt. Die Spirometrie ist nach wie vor notwendig, um die Diagnose zu stellen. Die Symptomatik wird meist anhand des Scores im COPD Assessment Tests (CAT) beurteilt. Das Risiko für Exazerbationen wird anhand der Exazerbationsrate im vergangenen Jahr bewer- tet. Eine Exazerbation liegt nach der neuen Leitlinie vor, wenn Patient und/oder Arzt eine Extratherapie als notwendig erachten.

\section{Algorithmus zur Therapieeskalation} Die zweite große Neuerung betrifft die Therapieeskalation innerhalb der Gruppen A bis D. Bei wenigen Symptomen (CAT-Score < 10) und maximal einer Exazerbation ohne Krankenhauseinweisung im vergangenen Jahr (Gruppe A) sollte ein Bronchodilatator eingesetzt werden.

Bei Patienten mit stärkeren Symptomen mit CAT-Score $\geq 10$ (Gruppe B) wird ein lang wirksamer Bronchodilatator (lang wirksames Beta-2-Mimetikum, LABA oder lang wirksamer Muskarinrezeptor-Antagonist, LAMA) als Monotherapie und bei persistierenden Symptomen deren Kombination empfohlen.

Patienten mit wenig Symptomen (CATScore $<10$ ), aber mindestens zwei Exazerbationen im vergangenen Jahr oder min-

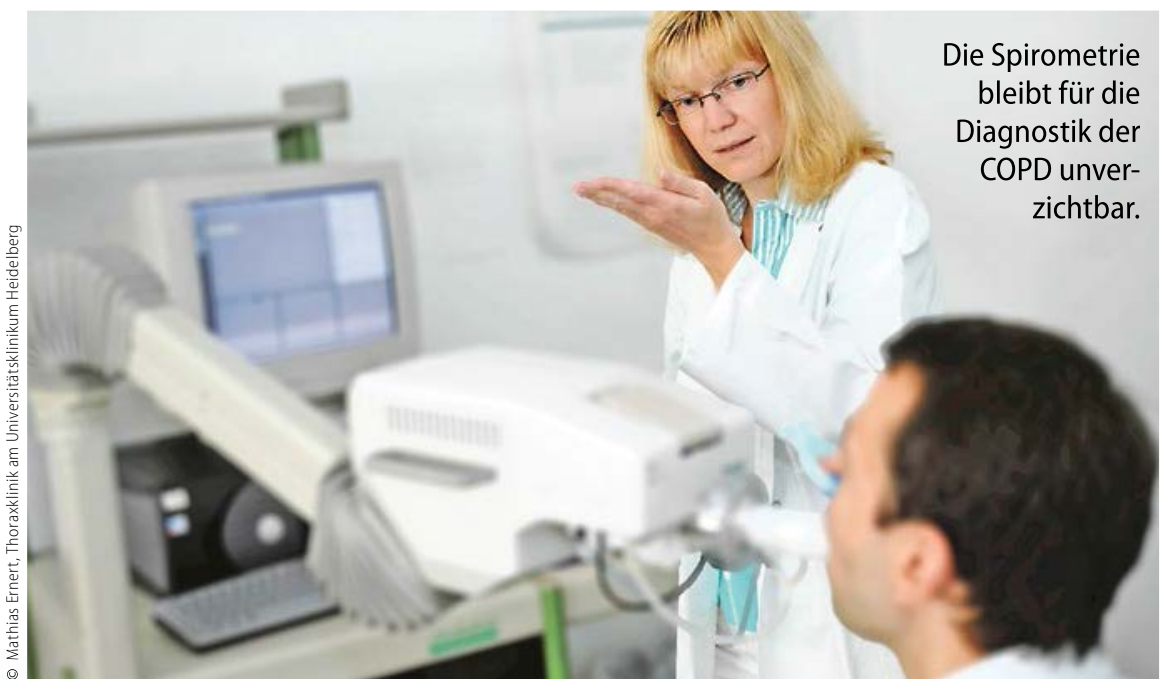

destens einer Exazerbation mit Krankenhauseinweisung (GOLD C), sollte bevorzugt ein LAMA verordnet werden. Bei weiteren Exazerbationen sollte eine LAMA/LABA-Kombitherapie erfolgen oder alternativ eine LABA/ICS-Kombination.

Bei erheblichen Symptomen (CATScore $\geq 10$ ) und häufigen Exazerbationen (Gruppe D) sollte man gleich eine LAMA/LABA Kombination einsetzen und bei weiteren Exazerbationen auf eine Tripletherapie mit LAMA/LABA/ICS umsteigen. Kommt es unter dieser Therapie $\mathrm{zu}$ weiteren Exazerbationen, kann zusätzlich Roflumilast (Daxas ${ }^{\circledast}$ ) gegeben werden, sofern das FEV $1<50 \%$ des Sollwertes beträgt und der Patient eine chronische Bronchitis hat.

\section{Medikamentöse Optionen}

Optionen aus der Klasse der LAMA sind: Tiotropium (Spiriva), Glycopyrronium (Seebri $^{\circledast}$ Breezhaler $^{\circledast}$ ), Umeclidinium (Incruse $^{\circledast}$ Ellipta $^{\circledast}$ ) und Aclidinium (Bretaris ${ }^{\circledast}$ Genuair $^{\circledast}$, Eklira ${ }^{\circledR}$ Genuair $^{\circledast}$ ). Alternativen sind die LABA Formoterol, Salmeterol, Indacaterol (Onbrez ${ }^{\circledR}$ Breezhaler $^{\circledR}$ ) und Olodaterol (Striverdi ${ }^{\oplus}$ Respimat $^{\oplus}$ ).

Es stehen mehrere LAMA/LABA-Fixkombinationen zur Verfügung: Indacaterol/Glycopyrronium (Ultibro ${ }^{\circledR}$ Breezhaler ${ }^{\oplus}$, Ulunar $^{\circledast}$ Breezhaler ${ }^{\circledast}$ ), Umeclidinium/ Vilanterol (Anoro ${ }^{\oplus}$ Ellipta $^{\otimes}$ ) und Tiotropium/Olodaterol (Spiolto ${ }^{\oplus}$ Respimat $^{\circledast}$ ) sowie Aclidinium/Formoterol (Brimica ${ }^{\circledR}$ Genuair ${ }^{\circledast}$, Duaklir ${ }^{\circledast}$ Genuair $^{\circledast}$ ).

Fixkombis mit LABA plus ICS sind: Formoterol/Budesonid (Symbicort ${ }^{\circledR}$ ), Salmeterol/Fluticason (Viani ${ }^{\circledast}$, Atmadisc ${ }^{\circledast}$ ) und Beclometason/Formoterol (Foster ${ }^{\circledR}$ ).

Bald wird es wohl auch eine erste Dreierfixkombination mit LAMA, LABA und ICS geben: Der Ausschuss für Humanarzneimittel der EMA hat vor Kurzem eine positive Stellungnahme für die Formoterol/Glycopyrronium/Beclometason-Fixkombination veröffentlicht. 